Korean J Ophthalmol 2021:35(5):413-414 https://doi.org/10.3341/kjo.2021.0051

\section{Optic Neuritis Presenting in Hashimo- to's Encephalopathy: A Case Report}

\section{Dear Editor,}

Hashimoto's encephalopathy is a rare clinical condition first described by Lord Brain, presenting with unexplained encephalopathy with detectable antithyroid antibodies [1]. Brain imaging of these patients display varied findings from normal to diversified appearance, including ischemic lesions, white matter demyelination, and focal vasogenic edema [2]. Characteristically, these patients usually have high titers of anti-thyroid peroxidase antibody. The disease belongs to the spectrum of the autoimmune disorders [3], which may share some characteristics of other diseases that accompany optic neuritis. The authors present a rare case of Hashimoto's encephalopathy accompanying optic neuritis.

A 32-year-old male was referred to the ophthalmic clinic with a 1-week history of painless vision loss in right eye. The patient was administered to the department of neurology for the evaluation of the dysarthria as well as right sided weakness of the upper and lower limb which started 2 days ago. On his blood test, the level of T3 and T4 was intact $(0.76 \mathrm{ng} / \mathrm{mL}$ and $1.22 \mathrm{ng} / \mathrm{dL}$, respectively), although thyroid stimulating hormone level was slightly decreased to $0.316 \mu \mathrm{IU} / \mathrm{mL}$. The levels of anti-thyroid peroxidase antibody (normal range, 0-60 U/mL) and anti-thyroglobulin antibody (normal range, $0-60 \mathrm{U} / \mathrm{mL}$ ) were proved to be high (3,411 and $487 \mathrm{U} / \mathrm{mL}$, respectively) but anti-neuromyelitis optica antibody was negative. Spinal fluid analysis revealed increased levels of albumin but oligoclonal band was negative. Other results suggestive of autoimmune or infective diseases were negative. The magnetic resonance imaging (MRI) of the patient displayed multifocal patchy signal changes in both cerebrum, cerebellum, thalami and brain stem, including subcortical white and gray matter.

Received: April 15, 2021 Final revision: July 7, 2021

Accepted: July 15, 2021
The patient was diagnosed as Hashimoto's encephalopathy for these findings.

Initial ophthalmic examination revealed visual acuity of $8 / 200$ in the right and $20 / 20$ in the left eye with relative afferent pupillary defect of grade 2 in right eye. There was severe dyschromatopsia in right eye. Disc swelling, however, was not found on the fundus examination, and the optical coherence tomography displayed intact retinal nerve fiber layer (RNFL) thickness and retinal ganglion cell (RGC) layer of the both eyes (Fig. 1A, 1B). Humphrey visual field test (30-2 SITA standard test) revealed incongruous right hemianopsia (Fig. 1C), which corresponded to the high signal lesion of the left parietotemporal optic radiations found in the initial T2 flare MRI (Fig. 1D). Central scotoma of the visual field was found on the right eye. The patient was therefore diagnosed as the optic neuritis of the right eye. Intravenous steroid pulse therapy was started, with $1 \mathrm{~g}$ of methylprenisolone for 5 days.

After a week, the visual acuity of the right eye recovered to $20 / 200$ and the dyschromatopsia began to improve. Optical coherence tomography showed decreased thickness of the RNFL layer as well as RGC layer of the right eye (Fig. 1E, 1F). Visual field still showed incongruous pattern of right hemianopsia, but the scotoma showed decrease compared with that of a week ago (Fig. 1G). High signal lesions found in the T2 flare MRI were unchanged of the size and intensity compared with that of a week ago (Fig. 1H).

After a month, the visual acuity of the right eye recovered to $20 / 20$, and the relative afferent pupillary defect was equivocal. RNFL thickness was not decreased any further, but the RGC layer thickness showed more loss (Fig. 1I, 1J). Results of the visual field test as well as color vision tests were recovered to normal (Fig. 1K). High signal lesions of the T2 flare MRI decreased in size as well as in signal intensities (Fig. 1L).

The authors reported a rare case of Hashimoto's encephalopathy accompanying optic neuritis. Previous reported common causes of optic neuritis were neuromyelitis optica spectrum disorders, multiple sclerosis, acute disseminated encephalomyelitis, systemic lupus erythematosus, and infectious diseases. There have been no prior cases of optic neuritis associated with Hashimoto's encephalopathy. Pre- 

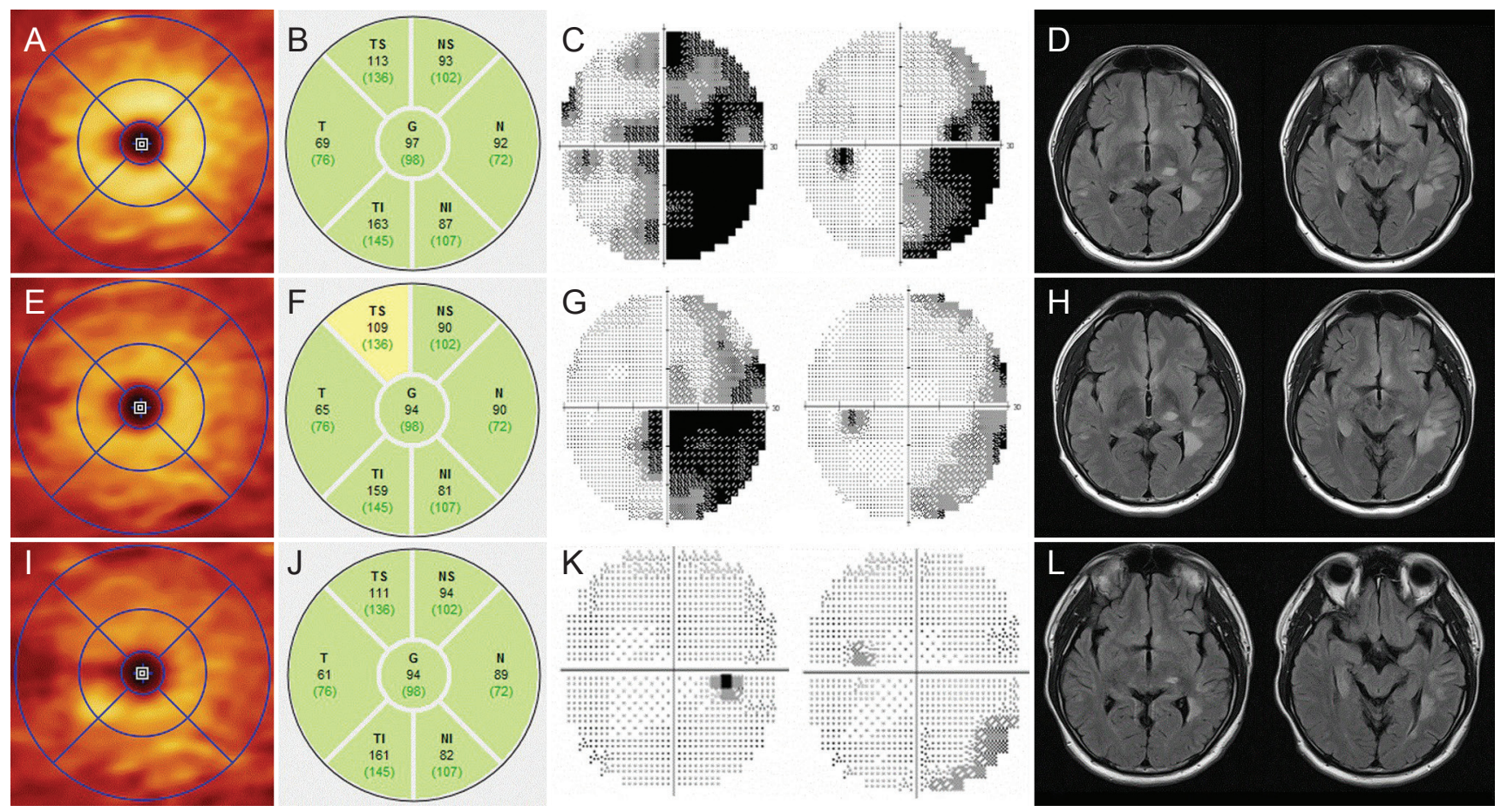

Fig. 1. A case of optic neuritis presenting in Hashimoto's encephalopathy. (A) Retinal ganglion cell layer thickness of the patient at initial presentation. (B) Summary of retinal nerve fiber layer thickness analysis of the patient at initial presentation. (C) Visual field of the patient at initial presentation. (D) T2 flare brain magnetic resonance imaging of the patient at initial presentation. (E) Retinal ganglion cell layer thickness of the patient after a week. (F) Summary of retinal nerve fiber layer thickness analysis of the patient after a week. (G) Visual field of the patient after a week. (H) T2 flare brain magnetic resonance imaging of the patient after a week. (I) Retinal ganglion cell layer thickness of the patient after a month. (J) Summary of retinal nerve fiber layer thickness analysis of the patient after a month. (K) Visual field of the patient after a month. (L) T2 flare brain magnetic resonance imaging of the patient after a month. Informed consent for publication of the clinical images was obtained from the patient. TS = temporal superior; $\mathrm{NS}=$ nasal superior; $\mathrm{T}=$ temporal; $\mathrm{G}=\mathrm{global}$; $\mathrm{N}=$ nasal; $\mathrm{TI}=$ temporal inferior; $\mathrm{NI}=$ nasal inferior.

viously known symptoms of Hashimoto's encephalitis include seizures, myoclonus, hallicunations and stroke-like episodes [4]. However, there have bene no other reports of such patients complaining of decreased visual functions. This report emphasizes that although the disease itself may be rare, Hashimoto's encephalopathy may be associated with optic neuritis. Therefore, when the patient with Hashimoto's encephalopathy complains of visual loss, optic neuritis should be considered in mind to avoid misdiagnosis.

Kyu Ho Chung, Jae Yong Park, Jin Choi

Department of Ophthalmology, Sanggye Paik Hospital, Inje University College of Medicine, Seoul, Korea

E-mail (JinChoi): jinchoi@paik.ac.kr

\section{Conflict of Interest}

No potential conflict of interest relevant to this article was reported.

\section{References}

1. Ferracci F, Moretto G, Candeago RM, et al. Antithyroid antibodies in the CSF: their role in the pathogenesis of Hashimoto's encephalopathy. Neurology 2003;60:712-4.

2. Kelley BP, Patel SC, Marin HL, et al. Autoimmune encephalitis: pathophysiology and imaging review of an overlooked diagnosis. AJNR Am J Neuroradiol 2017;38:1070-8.

3. Ong MS, Kohane IS, Cai T, et al. Population-level evidence for an autoimmune etiology of epilepsy. JAMA Neurol 2014;71:569-74.

4. Li J, Li F. Hashimoto's encephalopathy and seizure disorders. Front Neurol 2019;10:440. 